\title{
From Ramanujan to renormalization: the art of doing away with divergences and arriving at physical results
}

\author{
W. Bietenholz \\ Instituto de Ciencias Nucleares, Universidad Nacional Autónoma de México, \\ Apartado Postal 70-543, 04510 Ciudad de México, México.
}

Received 11 December 2020; accepted 5 February 2021

\begin{abstract}
A century ago, Srinivasa Ramanujan - the great self-taught Indian genius of mathematics - died, shortly after returning from Cambridge, UK, where he had collaborated with Godfrey Hardy. Ramanujan contributed numerous outstanding results to different branches of mathematics, like analysis and number theory, with a focus on special functions and series. Here we refer to apparently weird values which he assigned to two simple divergent series, $\sum_{n \geq 1} n$ and $\sum_{n \geq 1} n^{3}$. These values are sensible, however, as analytic continuations, which correspond to Riemann's $\zeta$-function. Moreover, they have applications in physics: we discuss the vacuum energy of the photon field, from which one can derive the Casimir force, which has been experimentally measured. We further discuss its interpretation, which remains controversial. This is a simple way to illustrate the concept of renormalization, which is vital in quantum field theory.
\end{abstract}

Keywords: Ramanujan summation; Casimir effect; renormalization; $\zeta$-function.

PACS: $01.60 .+\mathrm{q} ; 01.65 .+\mathrm{g} ; 03.70 .+\mathrm{k} ; 11.10 . \mathrm{Gh}$

DOI: https://doi.org/10.31349/RevMexFisE.18.020203

\section{Ramanujan's letter from 1913}

101 years ago, Srinivasa Ramanujan ${ }^{i}$ (1887-1920) passed away in Madras, at that time part of the British Empire (since 1996, this state capital in South-East India is named Chennai). He was one of the greatest geniuses ${ }^{i i}$ in the history of mathematics. One way to measure the impact of his work is through the number of mathematical terms that bear his name: the mathematical online-encyclopedia Wolfram Mathworld [1] documents 27 terms named after Ramanujan, and his name appears in a total of 205 items; in both respects, he is among the leading mathematicians of all times. ${ }^{i i i}$ This is particularly amazing since Ramanujan started to elaborate stunning equations with hardly any mathematical education, ${ }^{i v}$ and he died at the age of only 32 (younger than Mozart, for example).

Ramanujan was born in 1887 in a town called Erode, but at the age of 2, his mother took him to Madras, some $400 \mathrm{~km}$ away. In the early 20th century, he lived in extreme poverty, at the edge of starvation, but he discovered a multitude of important mathematical formulae,

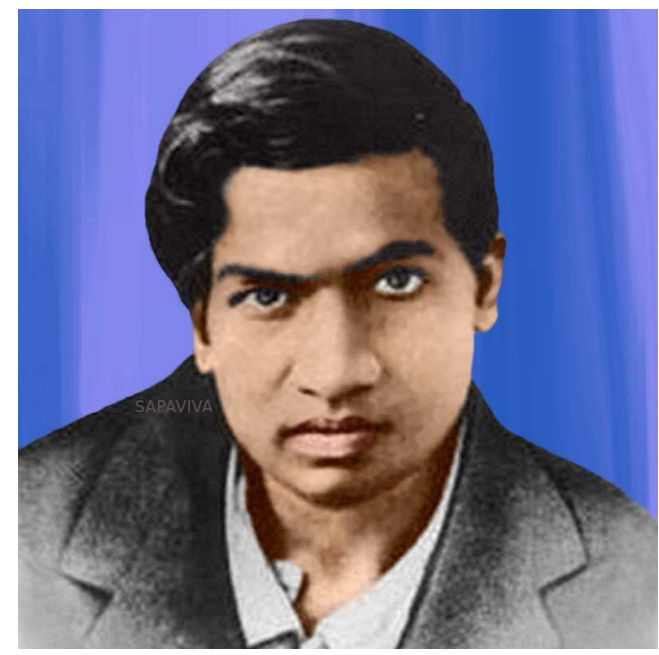

FIGURE 1. Srinivasa Ramanujan (1887-1920).

based on his incredible intuition - I tend to interpret it as a kind of "pattern recognition" (although it was not an automated process).

$i$ Phonetically, his last name could be written in Spanish as "Ramánuchan".

ii We understand the term "genius" as defined in the Cambridge Dictionary: a person who has a very great and rare natural ability or skill, especially in a particular area such as science or art.

iii To be explicit, if we rank mathematicians by the number of mathematical items named after them, Ramanujan is at position 6, following Euler (71), Gauss (48), Hilbert (33), Fermat (32), and Riemann (31), and followed by Cauchy (26), Dirichlet, Jacobi, Weierstraß (23 each), Euclid (22), and Poincaré (21). Regarding the number of mentionings in a Mathworld entry, Ramanujan is at position 18.

iv Ramanujan only obtained from a friend a library copy of a book by George Carr [2], which he studied intensively. It is a collection of formulae and theorems, with little explanation, written as an overview for students who are preparing for exams. 
In 1912, he began to send letters to British mathematicians, trying to attract attention to his discoveries; for a while without success. In January 1913, he finally wrote to Godfrey Hardy, a brilliant young mathematician at Trinity College of Cambridge University, who - together with his longterm collaborator John Littlewood - turned out to be the most influential British mathematician of the first half of the 20th century. They are credited for boosting British mathematics to the top level again after it had stayed behind the achievements in France and Germany during the 19th century. In particular, Hardy insisted on mathematical rigor, which was in total contrast to Ramanujan's intuitive style [3].

Unlike his colleagues, Hardy became aware of the enormous value of Ramanujan's results, although part of it had been known before, and some formulae were wrong - but the rest was groundbreaking [4]. Having received two letters with 120 remarkable equations, Hardy invited Ramanujan to Cambridge, which he accepted after some hesitation, and where he stayed from 1914 to 1919 , i.e., mostly during World War I. It was not easy for him to get used to the climate, lifestyle, and food. ${ }^{v}$ Moreover, he suffered from serious health problems; they had antecedents in his earlier life in India, and they lead to his decease one year after his return to Madras.

Despite appreciating his brilliance, Hardy urged him to take lectures (for instance, Ramanujan hardly knew anything about complex analysis), and in particular, he insisted on proofs, not just conjectures. That was not easily compatible with Ramanujan's mentality, but he published 32 high-impact papers during his 5 years in Cambridge, 7 of them together with Hardy [5]. In 1918 Ramanujan was elected as a Fellow of the Royal Society, as one of the youngest members in its

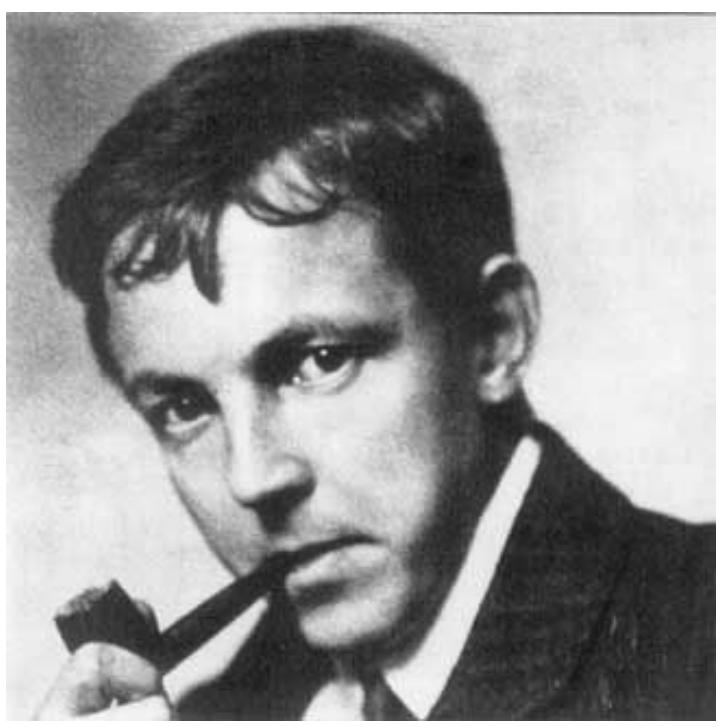

FIGURE 2. Godfrey Hardy (1877-1947). history, and half a year later, he also became a Fellow of the Cambridge Trinity College.

His investigations involved subjects, which had been considered intractable before, in particular a miraculous approximate formula for partitioning, which (surprisingly) involves the number $\pi$ [6]. Ramanujan traced this number in all kind of contexts; best known is a series that he postulated in Ref. [7] (along with a variety of other $\pi$-approximation formulae),

$$
\frac{1}{\pi}=\frac{\sqrt{8}}{99^{2}} \sum_{n \geq 0} \frac{(4 n) !}{\left(4^{n} n !\right)^{4}} \frac{1103+26390 n}{99^{4 n}} .
$$

It converges exponentially (despite the factor $(4 n)$ ! in the numerator); thus, it provides one of the fastest algorithms to compute $\pi$. If we truncate at $n_{\max }=0,1,2$, we obtain the corresponding approximation $\pi_{n_{\max }}$, which differs from the exact value of $\pi$ as

$$
\begin{aligned}
& \left|\pi-\pi_{0}\right| \simeq 7.6 \cdot 10^{-8}, \quad\left|\pi-\pi_{1}\right| \simeq 6.4 \cdot 10^{-16}, \\
& \left|\pi-\pi_{2}\right| \simeq 5.7 \cdot 10^{-24} .
\end{aligned}
$$

How Ramanujan arrived at such formulae is hard to know: Hardy later described it as a "process of mingled argument, intuition, and induction, of which he was entirely unable to give any coherent account" [8].

Here we are going to address a relatively simple subject, which Ramanujan mentioned in his first letter to Hardy [9], and which he had written down before in Chapter VI of his Second Notebook [10]. This letter contains two apparently weird formulae for divergent series,

$$
\begin{aligned}
& \sum_{n \geq 1}^{(\mathrm{R})} n=1+2+3+4+5+\cdots=-\frac{1}{12}, \\
& \sum_{n \geq 1}^{(\mathrm{R})} n^{3}=1+8+27+64+125+\cdots=\frac{1}{120},
\end{aligned}
$$

where the sums run from $n=1 \ldots \infty$, and the superscript (R) indicates "Ramanujan summation" [11]. These strange relations have fascinated generations of people; for instance, a discussion of Eq. (3) in YouTube [12], dated 2016, has over 2.4 million views and over 5000 quite controversial comments.

Of course, it is provocative to write these relations as straight equations, as Ramanujan did (without any superscript), but it fulfills the purpose of attracting attention and causing debate. Still, in the following, we are going to replace the symbol = by $\triangleq$, meaning "corresponds to" or "is associated with". In this sense, we are going to show that the

$v$ Being a devout Hindu, Ramanujan was a strict vegetarian, which was highly unusual in England at that time. 
fractional values on the right-hand side do have a meaning, not only as a mathematical peculiarity, but they can even be used to derive physical results.

Unlike other addressees of Ramanujan's letters, Hardy recognized the values of the Riemann $\zeta$-function or $p$-series. For $\operatorname{Re} z>1$, it is defined as

$$
\zeta(z)=\sum_{n \geq 1} \frac{1}{n^{z}}=\frac{1}{1^{z}}+\frac{1}{2^{z}}+\frac{1}{3^{z}}+\frac{1}{4^{z}}+\ldots .
$$

In 1739 Leonhard Euler had computed explicit expressions for $\zeta(2 n), n \in \mathbb{N}_{+}$, and later, he also conjectured a $\zeta$ functional relation [13]. More than a century later, in 1859, Bernhard Riemann established the analytic continuation of the $\zeta$-function to $\mathbb{C}-\{1\}$ [14], see Appendix C. In this sense, Hardy noticed that Ramanujan's results can be interpreted as $\zeta(-1)$ and $\zeta(-3)$ (although these values are not explicitly given in Ref. [14]).

Riemann was a leading mathematician of the 19th century, and of all times, cf. footnote [iii]. Like Ramanujan, he lived his youth in harsh poverty, until he was appointed to a post in Göttingen, on Carl Friedrich Gauss's recommendation. Another analogy is that he soon suffered from health problems. Hoping that a warmer climate might help against his tuberculosis [15] (which was also among Ramanujan's diseases [3]), he spent extended periods in Italy, where he died in 1866, at the age of 39 .

Differences from Ramanujan's life are that Riemann had access to education at leading mathematical institutes, in

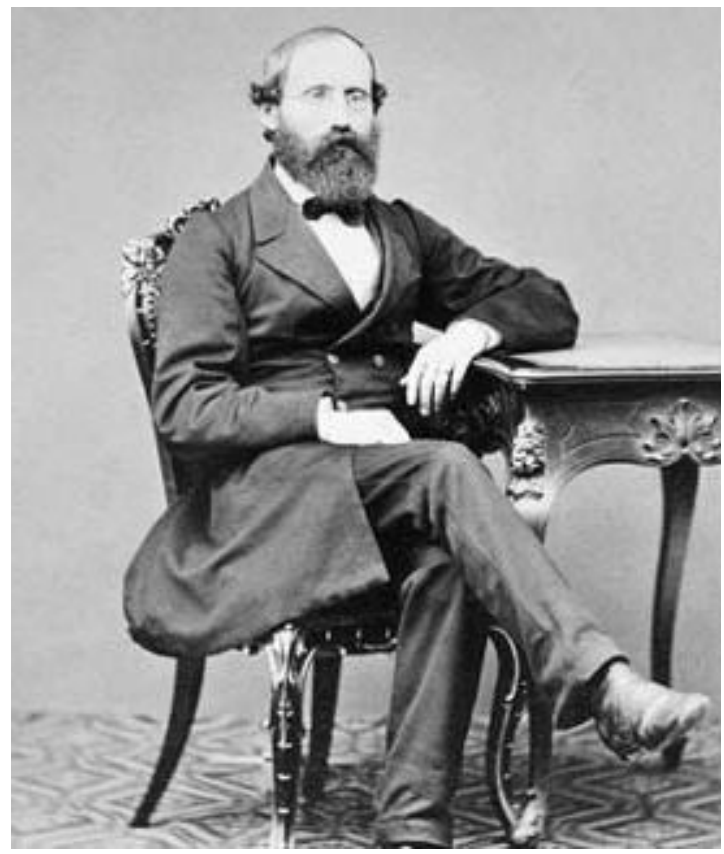

FIGURE 3. Bernhard Riemann (1826-66).
Göttingen and Berlin, and that he published his results only after elaborating rigorous proofs. His publications had an enormous impact, but only Ref. [14] deals with number theory. There he discussed the density of prime numbers, ${ }^{v i}$ and it was in this context that he postulated the analytic continuation of the $\zeta$-function; the crucial functional equation is displayed in Appendix C. In contrast to Ramanujan, Riemann was an expert on complex analysis. Presumably, he had handwritten notes with many more important results, but after his sudden death, his house-cleaner burned part of these notes, until some mathematicians managed to stop her [16].

Ramanujan did not provide an actual derivation of formulae (3) and (4), but in the first case, he assigned - in Ref. [10] and also his first letter to Hardy - a value to another divergent series, as an intermediate step to arrive at relation (3). That series corresponds to a special case of Dirichlet's $\eta$-function, or alternating $\zeta$-function

$$
\eta(z)=\sum_{n \geq 1} \frac{(-1)^{n-1}}{n^{z}}=\frac{1}{1^{z}}-\frac{1}{2^{z}}+\frac{1}{3^{z}}-\frac{1}{4^{z}} \ldots
$$

which converges for $\operatorname{Re} z>0$. At $\operatorname{Re} z>1$, we obtain

$$
\begin{aligned}
\zeta(z)-\eta(z) & =2 \sum_{n \geq 1} \frac{1}{(2 n)^{z}}=2^{1-z} \zeta(z), \\
\zeta(z) & =\frac{1}{1-2^{1-z}} \eta(z) .
\end{aligned}
$$

The latter defines $\zeta(z)$ in the domain $\operatorname{Re} z>0 \wedge z \neq 1$.

In particular, Ramanujan wrote down its continuation to [10]

$$
\begin{aligned}
\mathcal{E} & :=\eta(-1)=\sum_{n \geq 1}(-1)^{n-1} n \\
& =1-2+3-4 \ldots \stackrel{\wedge}{=} .
\end{aligned}
$$

We are going to confirm this value, and follow his path to relations (3) and (4), which we finally apply to physics, in particular to the Casimir effect.

\section{Heuristic derivation of $\sum_{n \geq 1} n \stackrel{\wedge}{=}-1 / 12$}

Series have both fascinated and confused mathematicians over and over again, throughout history. The famous "paradox" by Zeno, which describes a race between Achilles and a tortoise (and further "paradoxes" of a similar style), caused a deep crisis in the mathematics of Ancient Greece (see, e.g., Ref. [17]), because the concept of convergent series - in this case, a geometrical series - had not yet been understood.

$v i$ This is another field of common interest of these two geniuses: later, Ramanujan proposed his own formula for the prime number density, which is, however, not as accurate as he had expected. 
Here we just take the familiar geometrical series as the point of departure. For $|z|<1$, we trivially obtain

$$
\begin{aligned}
G(z) & =\sum_{n \geq 0} z^{n}=1+z+z^{2}+z^{3} \ldots \\
& =1+z G(z) \Rightarrow G(z)=\frac{1}{1-z} .
\end{aligned}
$$

The series converges only for $|z|<1$, but the final function $G(z)$ is defined all over $\mathbb{C}-\{1\}$. Moreover, the complex function $G(z)$ is holomorphic (or complex analytic, i.e., complex differentiable) ${ }^{\text {vii }}$ in $\mathbb{C}-\{1\}$, and therefore meromorphic in $\mathbb{C}$, which implies that its analytic continuation from the disk $|z|<1$ to $\mathbb{C}-\{1\}$ is unique, cf. Appendix $\mathrm{C}$.

This allows us to define Grandi's series

$$
\mathcal{G}=1-1+1-1+1-1 \cdots=\sum_{n \geq 0}(-1)^{n}
$$

by means of analytic continuation,

$$
\left.\mathcal{G} \triangleq G(z)\right|_{z=-1}=\frac{1}{2} .
$$

We can readily extend this scheme to the $\eta$-function in eqs. (6), (8). To this end, we first return to safe grounds, i.e., to $|z|<1$, where

$$
\begin{aligned}
G(z)^{2} & =1+2 z+3 z^{2}+4 z^{3} \ldots \\
& =\sum_{n \geq 1} n z^{n-1}=G^{\prime}(z)=\frac{1}{(1-z)^{2}} .
\end{aligned}
$$

The function $G(z)^{2}$ is holomorphic as well, again with a (unique) analytic continuation to $\mathbb{C}-\{1\}$. This implies in particular

$$
\mathcal{E}=\left.\sum_{n \geq 1}(-1)^{n-1} n \triangleq G(z)^{2}\right|_{z=-1}=\mathcal{G}^{2}=\frac{1}{4},
$$

which coincides with Ramanujan's result (8).

However, this is not yet what we need in order to assign a value to the notorious series, which we denote as

$$
\mathcal{R}:=\sum_{n \geq 1} n=1+2+3+4+5+\ldots .
$$

It would correspond to $\left.G(z)^{2}\right|_{z=1}$, but $z=1$ is just the point where this function has its double pole. Following Ramanujan's line of thought [10], we proceed by introducing another series

$$
\begin{aligned}
R_{1}(z) & =1-2 z+3 z^{2}-4 z^{3}+5 z^{4} \ldots \\
& =\sum_{n \geq 1} n(-z)^{n-1},
\end{aligned}
$$

which also converges at $|z|<1$, and we formally obtain $\mathcal{R} \triangleq R_{1}(-1)$. $^{\text {viii }}$ Again, we refer to the safe region, i.e., to the disk $|z|<1$, where we take the difference

$$
\begin{aligned}
G(z)^{2}-R_{1}(z) & =4 z\left(1+2 z^{2}+3 z^{4}+\ldots\right) \\
& =4 z \sum_{n \geq 1} n z^{2(n-1)} .
\end{aligned}
$$

This operation can only be justified inside the convergence disk, but once it is carried out, taking the limit $z \rightarrow-1$ on both sides leads to

$$
\begin{aligned}
\lim _{z \rightarrow-1}\left[G(z)^{2}-R_{1}(z)\right] & =\frac{1}{4}-\mathcal{R} \stackrel{\wedge}{=}-4 \mathcal{R} \\
& \Rightarrow \mathcal{R} \triangleq-\frac{1}{12} .
\end{aligned}
$$

Thus Ramanujan removed the divergence in a controlled manner, which leaves an unambiguous finite value, and Hardy noticed that this assignment corresponds to $\mathcal{R}=$ $\zeta(-1)$. In Appendix B, we will discuss what has been going on here.

Alternatively we could invoke Eq. (7) and consider the divergent series $\mathcal{C}=1+1+1+1+1 \ldots$ The limit $z \rightarrow 0$ implies

$$
\mathcal{C} \triangleq \zeta(0) \triangleq-\eta(0) \triangleq-\mathcal{G}=-\frac{1}{2},
$$

a value which is also given in Ramanujan's Second Notebook [10]. When we even insert $z=-1$ in Eq. (7), we arrive again at

$$
\mathcal{R} \triangleq-\frac{1}{3} \mathcal{E} \triangleq-\frac{1}{12}=\zeta(-1)
$$

So far, this may look like a mathematical playground, but in the next section, we are going to apply this result to a physical toy model, where it leads to sensible results. In Sec. 4, we proceed to a setting, which refers to physical phenomenology; for that purpose, we will need relation (4), which corresponds to $\zeta(-3)$.

vii We recall that this is a powerful property, which guarantees that the function has derivatives of any order in its domain of holomorphy, and that it coincides with its power series. Moreover, since $G^{\prime}(z) \neq 0$, it is also conformal, i.e., angle conserving: if we interpret the function $G(z)$ as a map $\mathbb{C} \rightarrow \mathbb{C}$, and consider two curves $\gamma_{1}(z), \gamma_{2}(z)$, which intersect in $z_{0}$ with a certain angle, then the maps of these curves intersect in $G\left(z_{0}\right)$ with the same angle.

viii The reason for the notation with an index 1 will become clear in Appendix B. 


\section{Casimir effect on a line}

In this section and beyond, we are going to deal with quantum field theory. General introductions can be found in a number of textbooks, such as Refs. [18] (and a popular science description is given in Ref. [19]), but in order to follow the derivations in Secs. 3 and 4, only very little knowledge about it is required. Our notation implicitly refers to the functional integral formulation, where the fields are functions of space and time variables, with values which can, for instance, be real numbers (then it is a neutral scalar field, as in this section), or vectors (as in Sec. 4) ${ }^{i x}$. In general, all field configurations - i.e., all possible values in each space-time point are integrated to obtain expectation values of observables.

Here, however, we are only concerned with the ground state contributions of free fields. For a neutral scalar field, we can imagine an (infinite) set of coupled harmonic oscillators, one at each space point. A Fourier transform yields oscillators for all possible frequencies. A priori, these frequencies are not restricted, so if we sum up their ground state contributions to the vacuum energy density, the result diverges.

We are going to be confronted with these ultraviolet (UV) divergences: they require a regularization, i.e., a mathematical modification which makes such sums (or integrals) finite, enabling calculations. In the end, we want to remove the regularization; hence, we aim at a cancelation of the UV divergences. This can often be achieved by subtracting divergent terms, so-called counterterms, which correspond to some limit; without taking that limit, a finite quantity remains. This procedure is known as a renormalization: it should lead to finite results for the physical quantities, which do not depend on the regularization that has been chosen (if suitable conditions are fulfilled).

The concepts, which we have sketched here in an abstract form, are going to be illuminated by the presentation of simple examples.

To this end, we address an effect, which was theoretically predicted by the Dutch physicists Hendrik Casimir and Dirk Polder in 1947/8 [20]. In particular, we follow the perspective that Casimir adopted a little later [21], inspired by a discussion with Niels Bohr.

As a toy model, we consider a free, neutral, massless scalar field on a line, $\phi(t, x) \in \mathbb{R}, x \in \mathbb{R}$. At the points $x=0$ and $x=d>0$ we apply Dirichlet boundaries, which force the field to vanish, i.e. $\phi(t, 0)=\phi(t, d)=0$. In this interval the field configurations can be Fourier decomposed into standing waves with wavenumbers $k_{n}=n \pi / d$, $n=1,2,3 \ldots$ (such that $\sin \left(k_{n} d\right)=0$ ). In natural units ( $\hbar=c=1$ ), they contribute $k_{n} / 2$ to the ground state energy.
For the vacuum energy density in this interval, we formally obtain

$$
\rho_{d}=\frac{1}{2 d} \sum_{n \geq 1} k_{n}=\frac{\pi}{2 d^{2}} \sum_{n \geq 1} n,
$$

where we encounter the divergent term $\mathcal{R}$, to which Ramanujan assigned the value $-1 / 12$. We are now going to illustrate - in a physical framework - why, and in which sense, this value is indeed meaningful.

As usual in quantum field theory, we first need a regularization (as we mentioned before), but it doesn't need to be fully specified. We regularize $\rho_{d}$ by performing a substitution

$$
n \rightarrow f(n)=n r(n / \Lambda d), \quad \text { with } \quad r(0)=1,
$$

where $f$ and $r$ are smooth functions on $\mathbb{R}_{0}^{+}$(an infinite number of times continuously differentiable), and $\Lambda$ is an energy cutoff. If we remove it, $\Lambda \rightarrow \infty$, we recover the term before regularization. At finite $\Lambda$, we require

$$
\lim _{x \rightarrow+\infty} f(x)=0, \quad \lim _{x \rightarrow+\infty} f^{(k)}(x)=0,
$$

where $f^{(k)}$ is any odd derivative $(k=1,3,5, \ldots)$.

A simple example of $f(n)$ in Eq. (21) is the heat kernel regularization, where the function $r$ is exponential, $f(n)=$ $n \exp (-n / \Lambda d)$, which leads to a geometrical series,

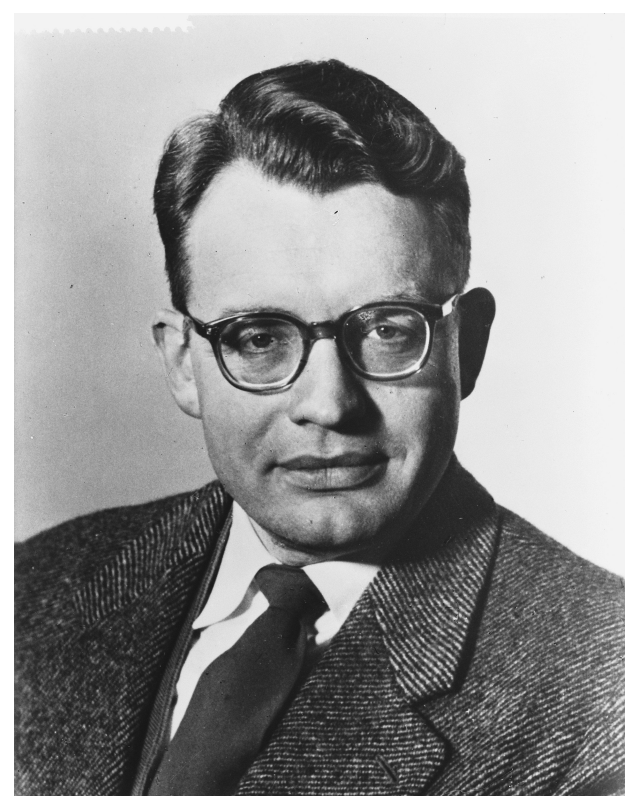

FIGURE 4. Hendrik Casimir (1909-2000).

$i x$ Alternatively, in the canonical formalism the fields are operator-valued. 


$$
\begin{aligned}
\sum_{n \geq 1} n e^{-n / d \Lambda} & =\Lambda^{2} d \frac{\partial}{\partial \Lambda} \sum_{n \geq 0} e^{-n / \Lambda d} \\
& =\Lambda^{2} d \frac{\partial}{\partial \Lambda} \frac{1}{1-\exp (-1 / \Lambda d)} \\
& =(\Lambda d)^{2}-\frac{1}{12}+\mathcal{O}\left(\frac{1}{\Lambda d}\right) .
\end{aligned}
$$

We already see $\mathcal{R}=-1 / 12$ popping up, but we want to proceed to a broader perspective, which generalizes the regularization, and which also clarifies the rôle of the UV divergent term, along the lines of Ref. [21].

In an infinite interval, $d \rightarrow \infty$, the formal term (20) for the energy density turns into a momentum integral. We expand the difference $\rho_{d}-\rho_{\infty}$, at the regularized level, by means of the Euler-Maclaurin formula,

$$
\begin{aligned}
\sum_{n=1}^{N} f(n) & -\int_{0}^{N} f(x) d x=\frac{f(N)-f(0)}{2} \\
& +\sum_{j \geq 1} \frac{B_{2 j}}{(2 j) !}\left(f^{(2 j-1)}(N)-f^{(2 j-1)}(0)\right)
\end{aligned}
$$

where $N \in \mathbb{N}_{+}$. A finite number $N$ represents another component of the UV regularization: in this case, we sum over $k_{n}$ only up to $k_{n, \max }=N \pi / d$.

The powerful formula (24) was independently derived by Euler and by Colin Maclaurin around 1735. It is very useful in field theory, in particular when dealing with finite temperature or finite-size effects. Since we assume the function $f$ to be smooth and to fulfill the condition (22), this series converges both at finite $N$ and in the limit $N \rightarrow \infty$. $^{x}$ The coefficients in the last term are the Bernoulli numbers, ${ }^{x i}$ which can be defined in a way related to Eq. (23),

$$
\frac{x}{1-e^{-x}}=\sum_{k \geq 0} B_{k} \frac{x^{k}}{k !} .
$$

This yields $B_{0}=1, B_{1}=1 / 2, B_{2}=1 / 6, B_{4}=-1 / 30$, $B_{3}=B_{5}=B_{7}=\cdots=0,{ }^{x i i}\left(B_{6}, B_{8} \ldots\right.$ do not vanish, but we won't need them).

We insert in Eq. (24) a function $f$ which fulfills the conditions (21) and (22), and we take the UV limit in two steps: first, we let $N \rightarrow \infty$; due to Eq. (22) all contributions vanish in this limit. As for the terms at $x=0$, we note that $f(0)=0$, $f^{\prime}(0)=r(0)=1, f^{(k)}(0)=\mathcal{O}\left((\Lambda d)^{1-k}\right), k=2,3, \ldots$, hence the second step of the UV limit, $\Lambda \rightarrow \infty$, leads to

$$
\rho_{d}-\rho_{\infty}=\frac{\pi}{2 d^{2}}\left(-\frac{1}{2} B_{2}\right)=-\frac{\pi}{24 d^{2}},
$$

where $\mathcal{R}=-B_{2} / 2=-1 / 12$ is crucial, in agreement with Eq. (23). We recover Ramanujan's assignment of a finite value to the divergent series in Eq. (20), i.e., Ramanujan summation corresponds to the subtraction of the infinite-volume limit of the vacuum energy density. This density diverges both in a finite and infinite interval, but the difference, i.e. its finite-size effect, is finite and well-defined. The elimination, or isolation, of a UV divergent term $\left((\Lambda d)^{2}\right.$ in Eq. (23)), in order to deal with finite differences, is the basic idea of renormalization. In field-theoretic jargon, we have subtracted the counterterm $\rho_{\infty}$, which cancels the divergence in the series (20).

Interestingly, this recipe matches exactly the relation

$$
\zeta(-1)=\sum_{n \geq 1}^{(\mathrm{R})} n=-\frac{1}{2} B_{2}=-\frac{1}{12},
$$

applied to Eq. (20). We recall that the superscript (R) means Ramanujan summation; its general properties are defined and explored in Ref. [11]. For this article, it is sufficient to point out that for a series of the form $\sum_{n \geq 1}^{(\mathrm{R})} n^{k}, k \in \mathbb{N}$, the Ramanujan summation coincides with the $\zeta$-function $\zeta(-k)$ (defined by analytic continuation), see Appendix C. It further corresponds to the finite term in the Euler-Maclaurin expansion of the difference

$$
\lim _{N \rightarrow \infty}\left(\sum_{n=1}^{N} n^{k}-\int_{0}^{N} x^{k} d x\right),
$$

which can be read off from Eq. (24), and which generalizes Eq. (27) to

$$
\zeta(-k)=\sum_{n \geq 1}^{(\mathrm{R})} n^{k}=-\frac{B_{k+1}}{k+1}, \quad k \in \mathbb{N} .
$$

The question remains: beyond the satisfaction of deducing a finite result, why are we interested in this difference?

\footnotetext{
$x$ If we truncated this expansion at some odd integer $J / 2$, such that we deal with $\sum_{j=1}^{J / 2} \ldots$, then there is a remainder term $R_{J}$ on the right-hand side. It can be estimated as $\left|R_{J}\right| \leq 2 \zeta(J) \int_{1}^{N} d x\left|f^{(J)}(x)\right| /(2 \pi)^{J}$ [22], hence, the above assumptions imply $\lim _{J \rightarrow+\infty} R_{J}=0$.

xi Bernoulli numbers were a particular passion of Ramanujan, who had certainly read about them in Ref. [2]. His very first paper discussed their properties [23]. For instance, he showed that the denominators of $B_{2}, B_{4}, B_{6}, B_{8} \ldots$ (in lowest terms) all contain the prime factors 2 and 3 exactly once.

xii This becomes obvious if we define $g(x)=x /\left(1-e^{-x}\right)$ and compute $g(x)-g(-x)=x$.
} 

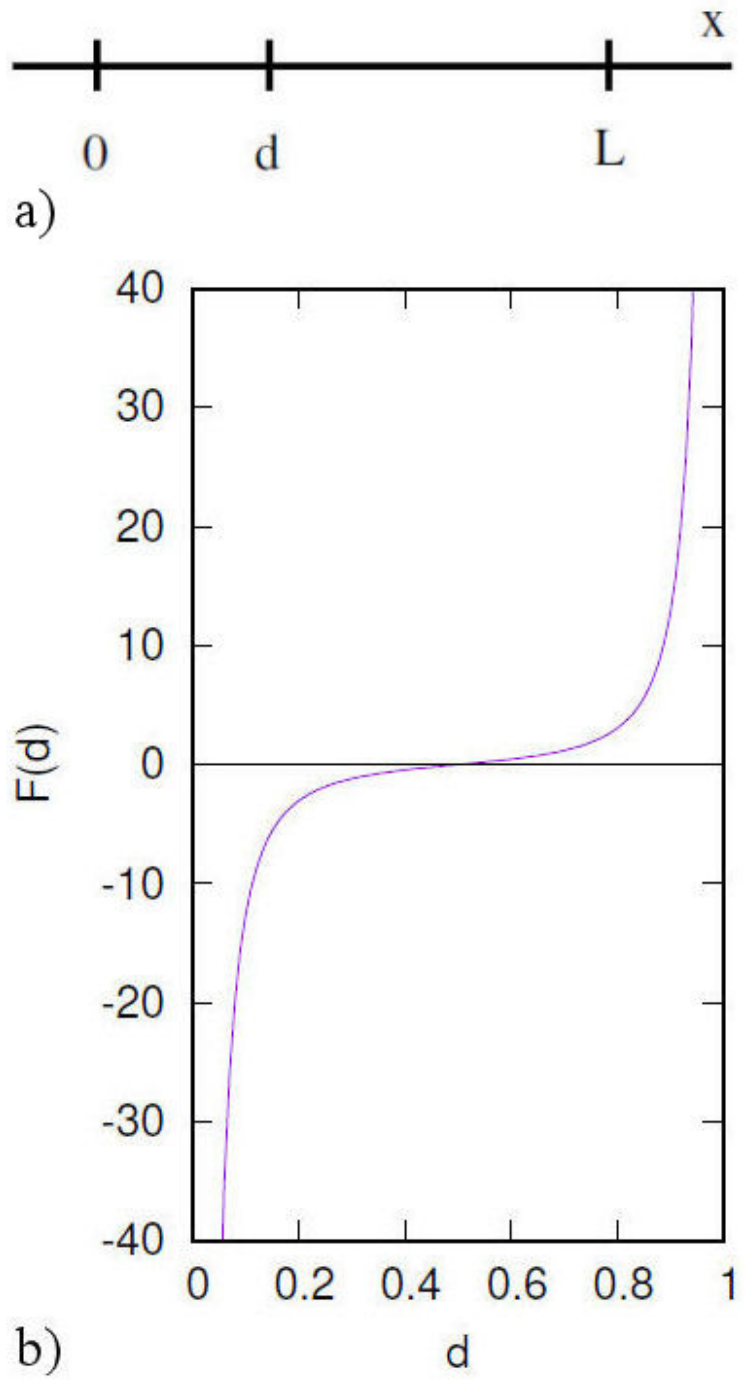

FIGURE 5. a) Setting for the Casimir effect on a line: we apply Dirichlet boundaries at the positions $0, d$, and $L$, with $0<d<L$. b) The Casimir force $F(d)$, which acts on the "piston" at $d$ according to Eq. (30), in units such that $L=1$.

What is its physical meaning? One is tempted to reply: the change of the vacuum energy, as a function of $d$, implies a force between the Dirichlet boundaries, and the counterterm can be subtracted since it does not depend on $d$, so it does not contribute to this force. However, there is still a caveat, which is often ignored: the boundaries could also affect the energy outside the interval $[0, d]$. That could contribute to the force between the boundaries, so we have to be careful.

A sound approach introduces three Dirichlet boundaries, at the points $0, d, L$, with $0<d<L$, see Fig. 5a).

The idea is to keep the extreme boundaries at 0 and $L$ fixed, while the one at $d$ is a variable "piston". In this way, the energy outside the interval $[0, L]$ remains constant, while the energy inside this interval can be computed explicitly, so everything is under control. From Eq. (26), we obtain the total vacuum energy

$$
\begin{aligned}
E(d) & =-\frac{\pi}{24 d}-\frac{\pi}{24(L-d)}+E_{\mathrm{out}} \\
& =-\frac{\pi L}{24 d(L-d)}+E_{\mathrm{out}} .
\end{aligned}
$$

The term $E_{\text {out }}$, which represents the energy outside the interval $[0, L]$, is divergent, but it does not depend on $d$. Generally a force is obtained as the negative gradient of the potential energy. In our 1-dimensional case, this operator reduces to the negative derivative with respect to $d$ (the only variable involved). Therefore the term $E_{\text {out }}$ is irrelevant for the force acting on the "piston" at $d$, which is obtained as

$$
F(d)=-E^{\prime}(d)=-\frac{\pi L}{24} \frac{L-2 d}{d^{2}(L-d)^{2}},
$$

and depicted in Fig. 5b). It is odd with respect to the center $d=L / 2$, and attractive towards the nearer fixed boundary, at 0 or $L$. Hence $d=L / 2$ is an unstable equilibrium position. In the case $L \gg d$, we obtain a force, which is attractive towards the boundary at $0, F(d) \simeq-\pi /\left(24 d^{2}\right)$, and which coincides with the 2-boundary picture of Eq. (26). Hence, in that picture, ignoring effects outside the interval $[0, d]$ is justified after all (varying $d$ does not change the energy in the half-line with $x>d$ ).

To summarize this section, we have renormalized the system by discarding an additive, infinite constant in the energy density, the counterterm $\rho_{\infty}$, which represents the infinitevolume limit. In order to compute the remaining finite term, a finite-size effect in this case, a regularization is needed. Then the Euler-Maclaurin formula can be applied, and by removing both UV cutoffs, $k_{n, \max }=N d / \pi \rightarrow \infty$ and $\Lambda \rightarrow \infty$, we arrive at the finite result (26). It does not depend on the choice of the regularizing function $f$, as long as the conditions (21) and (22) are fulfilled. This leads to finite values for the vacuum energy in the interval $[0, L]$, and for the force $F(d)$ in Eq. (30), which acts on the "piston".

\section{The Casimir force in 3-dimensional space}

We proceed to a realistic situation, which deals with the vacuum energy of the photon field in $(3+1)$-dimensional spacetime. The simplest setting is shown in Fig. 6; it involves two parallel, conducting plates, ${ }^{\text {xiii }}$ of the same rectangular shape and area $A$, separated by a short distance. ${ }^{\text {xiv }}$

xiii In theory, we assume perfect conductivity, this is what it takes to implement exact Dirichlet boundaries. The experiments have been performed with well-conducting metal plates, which provide a good approximation, cf. Sec.5.2. The generalization with respect to the dielectric constant was theoretically studied by Evgeny Lifshitz [24].

xiv Throughout this article, we refer to the standard scenario with static Dirichlet boundaries. The two-fold generalization of the Casimir effect with dynamical Robin boundaries is discussed for scalar fields in Refs. [25]. 


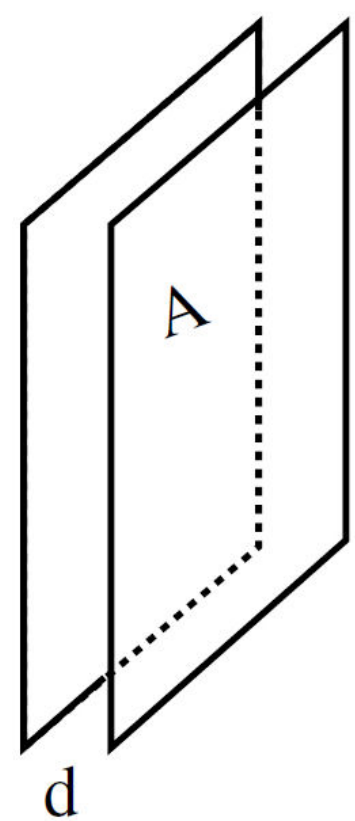

FIGURE 6. Setting for an experimental test of the Casimir effect: one measures the force between two parallel, conducting plates of area $A$, separated by a short distance $d$.

We rely on our experience from the 1-dimensional toy model to conjecture that it is sufficient to consider the energy $E(d)$ between the plates. This is appropriate when the area $A$ is large $(\sqrt{A} \gg d)$. The photon momentum components parallel to the plates - which we denote as $k_{1}, k_{2}$ - are treated as continuous. Hence we perform a discrete sum, in analogy to Eq. (20), only over the vertical component $k_{3}$, and the energy between the plates takes the form

$$
\begin{aligned}
E(d) & =A d \rho(d)=\frac{1}{2} A \frac{1}{(2 \pi)^{2}} \int d k_{1} d k_{2} \\
& \times \sum_{n \geq 0} 2 \sqrt{k_{1}^{2}+k_{2}^{2}+\left(\frac{\pi n}{d}\right)^{2}} \\
& =\frac{A}{2 \pi} \sum_{n \geq 0} \int_{0}^{\infty} d K K \sqrt{K^{2}+\left(\frac{\pi n}{d}\right)^{2}} \\
& =\left.\frac{A}{6 \pi} \sum_{n \geq 0}\left(K^{2}+\left[\frac{\pi n}{d}\right]^{2}\right)^{3 / 2}\right|_{0} ^{\infty},
\end{aligned}
$$

where we have inserted a factor 2 for the two photon polarization states.

Note that we have not regularized so far. If we do so and follow the procedure of Sec. 3, we can renormalize by subtracting the energy in the same volume but without plates,
$E_{\infty}=A d \rho_{\infty}{ }^{x v}$ In this difference, first, the UV contribution due to $K \rightarrow \infty$ cancels (a physical interpretation is that infinitesimally short wavelengths are not sensitive to the presence of boundaries at a finite distance). Regarding $K=0$, we apply the Euler-Maclaurin expansion (24) to

$$
\sum_{n \geq 1} \cdots-\int_{0}^{\infty} d k_{3} \ldots
$$

This corresponds to the Ramanujan summation over $n$, which we again express as a $\zeta$-function,

$$
\begin{aligned}
& \frac{E(d)}{A} \triangleq-\frac{1}{6 \pi} \frac{\pi^{3}}{d^{3}} \zeta(-3)=-\frac{\pi^{2}}{720 d^{3}}, \\
& \frac{F(d)}{A}=\frac{-E^{\prime}(d)}{A}=-\frac{\pi^{2}}{240 d^{4}} .
\end{aligned}
$$

We have used Eq. (28) and inserted $B_{4}=-1 / 30$, in agreement with Eq. (4). Although the sign of $B_{4}$ is opposite to $B_{2}$ (which we inserted in Eq. (26)), we obtain again an attractive force between the Dirichlet boundaries: note that there is another sign flip due to the integral over $K$, where the lower bound contributes.

The question arises of what magnitude this force takes for realistic sizes $A$ and $d$, and if such a force can be measured. The first conclusive experiment was achieved in 1997 by Steve Lamoreaux, who succeeded in measuring the Casimir force to $5 \%$ accuracy [26]. This was soon followed by Umar Mohideen and Anushree Roy [27], who took into account the corrections due to finite temperature, finite conductivity, and the roughness of the surfaces. In these experiments, the geometrical structure was a plate and a sphere, because of the difficulty in keeping two plates parallel to very high precision.

The first experiment to successfully measure the Casimir force between parallel plates, as sketched in Fig. 6, was carried out at the University of Padua, Italy, in 2002 [28], with $15 \%$ precision. That experiment used rectangular silicon stripes, covered with a chromium layer, of size $A=$ $1.9 \mathrm{~cm} \times 1.2 \mathrm{~mm}$, and their separation $d$ varied from $0.5 \mu \mathrm{m}$ to $3 \mu \mathrm{m}$. In order to compute the predicted force in Newton $(\mathrm{N})$, we have to insert a factor $\hbar c$ in Eq. (32), ${ }^{x v i}$ which leads to

$$
F(d) \simeq-1.3 \cdot 10^{-7} \mathrm{~N}\left(\frac{\mu \mathrm{m}}{d}\right)^{4} \frac{A}{\mathrm{~cm}^{2}} .
$$

Hence the predicted force in this experiment varied between $F \simeq-4.7 \cdot 10^{-7} \mathrm{~N}$ and $-3.7 \cdot 10^{-10} \mathrm{~N}$. Forces of this range are in fact measurable: for instance, Ref. [26] used a torsion

$x v$ To obtain $E_{\infty}$ we start from the term in the upper line of eq. (31), convert (in the large- $d$ limit) $\pi n / d$ to the continuous momentum component $k_{3}$, and $\sum_{n \geq 0}$ to $\frac{d}{\pi} \int_{0}^{\infty} d k_{3}$, which leads to $E_{\infty}=A d(2 \pi)^{-3} \int d^{3} k|\vec{k}|$.

$x v i$ We recall that we have been using natural units. This factor shows that we are dealing with a relativistic quantum effect. 
pendulum and laser interferometry, and Ref. [27] employed an atomic force microscope. In the experiment reported in Ref. [28], one of the parallel plates was the face of a cantilever beam, free to oscillate. The variation of the force was observed by measuring shifts in its resonator frequency, by means of a fiber-optic interferometer.

The precision in recent experiments is around, or below, $1 \%$.

\section{Concluding remarks}

We don't know what exactly Ramanujan had in mind when he introduced his summation of divergent series, which we now denote as Ramanujan summation, such as relations (3) and (4). In his letter to Hardy, he only documented one intermediate step, relation (13). In his Second Notebook [10], he additionally hinted at the continuation (17), and he mentioned the difference between summation and integration, which we also reviewed. This is a valid argument, and apparently, Ramanujan reinvented an equivalent form of the EulerMaclaurin formula (he did not use that term [10], nor does this formula appear in Ref. [2]).

A reason for the sparse documentation in Ramanujan's notebooks was - in addition to his intuitive way of thinking that he mostly worked on a slate and only wrote down final results on paper, which was valuable (in particular for him, who was living in poverty). It is also conceivable that he was influenced by Carr's telegram style [2], cf. footnote [iv]. In any case, his approach matches the analytic continuation of the $\zeta$ function, at least with respect to negative integer arguments, see Eq. (28). In fact, he also rediscovered the analytically continued $\Gamma$-function with values in $\mathbb{C}-\{0,-1,-2 \ldots\}$. He highlighted this idea in the introduction of his first letter to Hardy [9], unaware that this had been known before; in particular, Riemann had used it in Ref. [14].

These finite values for divergent series may look like a mathematical game, which is rather disconnected from reality. However, it is possible to establish consistent rules for the Ramanujan summation of divergent series by carefully dealing with properties like linearity and translation [11]. Moreover, we reviewed their striking application to physics, where they enable the prediction of a force, which has in fact been measured. The meaning of the Casimir effect will be discussed in the following two subsections.

\subsection{Is the electromagnetic vacuum energy density real?}

Numerous authors infer from the experimental observation of the Casimir force the existence of the vacuum energy of the photon field, $\rho_{\mathrm{vac}}$, as predicted by Quantum Electrodynamics (QED), e.g., Refs. [26-31]. As a typical quotation, Ref. [31] states that "the existence of zero-point vacuum fluctuations has been spectacularly demonstrated by the Casimir effect." However, it does not affect usual experiments, which only depend on energy differences, not on the additive constant $\rho_{\text {vac. }}$. Still, such an energy density throughout the Universe, known as Dark Energy, is indeed manifest since it affects the expansion of the Universe.

It corresponds to the Cosmological Constant in General Relativity: in its absence - which was generally assumed from the 1930s to the 1990s - the expansion of the Universe would be decelerated. However, at the very end of the 20th century, it was observed that the expansion is accelerated. ${ }^{x v i i}$ This is best described by a positive Cosmological Constant, which corresponds to a Dark Energy density of about $\rho_{\mathrm{DE}} \approx$ $(0.002 \mathrm{eV})^{4}$.

Unfortunately, this value is totally incompatible with the vacuum energy density $\rho_{\infty}$ that we discussed. First, $\rho_{\infty}$ seems to diverge, as we saw, but one might impose a UV cutoff in the integral $\int d^{3} k|\vec{k}|$, most naturally at the Planck energy. This leads to a finite value $\rho_{\text {Planck }}$, which is, however, much too large, $\rho_{\text {Planck }} / \rho_{\text {DE }}=\mathcal{O}\left(10^{121}\right)$.

People who still believe in supersymmetry could argue that in a perfectly supersymmetric world, the Dark Energy vanishes (since bosons and fermions appear in pairs of the same mass, and the fermionic ground state energy is negative, with the same absolute value [18]). However, even if supersymmetry exists, it has to be badly broken in our lowenergy world (otherwise particles like the "selectron" would have been observed), and the required extent of breaking still implies a Dark Energy density, which exceeds $\rho_{\mathrm{DE}}$ at least by a factor $\mathcal{O}\left(10^{60}\right)[30]$.

Hence any evidence for the existence of the QED photon field vacuum energy $\rho_{\text {vac }}$ would be puzzling. Albeit, Julian Schwinger et al. computed the Casimir using a source field technique, without any need to refer to $\rho_{\mathrm{vac}}$ [33]. Part of the literature concludes from that work that the Casimir experiments do not necessarily imply the reality of $\rho_{\mathrm{vac}}$, which could be welcome as a remedy against the disastrous discrepancy by 121 orders of magnitude. Thus the question remains whether or not relativistic quantum physics could be formulated without $\rho_{\mathrm{vac}}$. If $\rho_{\mathrm{vac}}$ exists, in the field-theoretic sense, one might wonder whether the frequency of a photon is affected when it passes through regions of different $\rho_{\mathrm{vac}}$, e.g., when it transversally passes through a Casimir cavity, similar to Bernoulli's Principle in fluid dynamics. Regarding its vertical motion, there is even a prediction that the speed of light could be affected [34].

If we wanted to construct a cavity between two conducting plates with $\rho_{\mathrm{DM}}=\left|\rho_{d}\right|=\pi^{2} /\left(720 d^{4}\right)$, we would need a separation of $d \approx 0.3 \mu \mathrm{m}$, which happens to be close to the minimal separation in the Padua experiment. 


\subsection{The nature of the Casimir force}

One could question what kind of force this really is. It does not seem to appear in the famous list of four forces, which can be described by gauge fields, nor does it match further interactions in the Standard Model of particle physics (Yukawa couplings and the Higgs field self-coupling). However, being an effect of the photon field, this force must ultimately be electromagnetic, although this is not explicit in the above discussion. From this perspective, it can be best described as a van der Waals force $e^{x v i i i}$ between the metal plates. This is the picture that Casimir and Polder originally had in mind [20].

So, do we have two equivalent descriptions? This seems puzzling again: in the van der Waals picture, the force depends on the value of the electromagnetic coupling constant $\alpha=e^{2} / 4 \pi \simeq 1 / 137$, which does not appear anywhere in the discussion based on the vacuum energy.

This point was analyzed in depth by Robert Jaffe and collaborators, see Ref. [36] for a summary. They conclude that the Casimir effect is a relativistic quantum force between electric charges and currents, i.e., a retarded van der Waals force, which does not require $\rho_{\mathrm{vac}}$. In Jaffe's own words, "Casimir effects can be formulated, and Casimir forces can be computed without reference to zero-point energies." Thus they contradict the paradigm in this field, but this issue remains controversial.

Reference [36] obtains a Casimir force, which does depend on $\alpha$, such that $F(\alpha=0)=0$, and $F(\alpha \simeq 1 / 137)$ is the physical strength. In this exceptional case, even the limit $\alpha \rightarrow \infty$ leads to a finite result: $F(\alpha \rightarrow \infty)$ just matches the force obtained from $\rho_{\text {vac }}$, which is often close to $F(\alpha \simeq 1 / 137)$. For instance, for copper plates separated by $0.5 \mu \mathrm{m}$ (which is experimentally realistic), the consideration with $\rho_{\mathrm{vac}}$ is a good approximation if $\alpha \gg 10^{-5}$ [36], which is easily accomplished by the phenomenological value.

A wide-spread objection against that point of view refers to examples, where the consideration based on $\rho_{\text {vac }}$ leads to a repulsive Casimir force [37], e.g., for specific parallelepipeds [38]. That feature is not easily encompassed by van der Waals forces. ${ }^{\text {xix }}$ For instance, Lamoreaux [26] writes: "the Casimir and van der Waals forces are quite different; the van der Waals force is always attractive, whereas the sign of the Casimir force is geometry dependent." Ref. [40] disagrees and assigns the repulsive result to the negligence of cutoff effects. In fact, an approach by Ricardo Cavalcanti [41], which is manifestly free of any cutoff dependence, only obtains attractive Casimir forces.
Jaffe and his collaborators insist that the physical Casimir force is always attractive $[36,40]$, and therefore compatible with the van der Waals picture. If this alternative to the paradigm - as expressed in Refs. [26-31] - is correct, then the approach that we reviewed is not the most precise one, but it is still in agreement with the experimental results.

Thus, returning to the title of Subsec. 5.1, doubts persist about the physical reality of $\rho_{\mathrm{vac}}$ as encoded in QED. There is a consensus, however, that we do not know how to theoretically derive the Dark Matter density $\rho_{\mathrm{DE}}$, and that we do not understand the enormous discrepancy from the vacuum energy predicted by quantum field theory.

So far, our discussion in this subsection focused on the Casimir effect due to QED, which was described in Sec. 4. In principle, such an effect also exists for other gauge fields, but only for QED it is simple and instructive, in particular, because the photon field does not self-interact. This is also the only case where the Casimir force is experimentally confirmed.

For instance, in Quantum Chromodynamics (QCD) - the gauge theory of the strong interaction - this effect is much less transparent because of the complicated self-interaction of the gluon field [18], which occurs since the QCD gauge group $\mathrm{SU}(3)$ is non-Abelian. ${ }^{x x}$ At low energy, its behavior is dominated by non-perturbative effects, which are hard to compute, and which induce an intricate vacuum structure. Studies in Euclidean space often focus on the rôle of instantons [42]. For a static quark-anti-quark pair (which is an idealization), a multipole expansion has been applied to estimate the Casimir force [43]. Another study [44] deals with the (restricted) Gribov-Zwanziger action.

Furthermore, there are numerous attempts to theoretically investigate the gravitational Casimir force, although this is a quantum effect, and we do not have any (fully satisfactory) theory of quantum gravity. Numerous papers refer to unusual gravitation theories; studies which are (roughly speaking) close to the framework of General Relativity include Refs. [45]. The question of whether an experimental demonstration of such an effect, with gravitational wave mirrors, would prove the existence of gravitons is discussed (and negatively answered) in Ref. [46].

\subsection{Further physical applications of Ramanujan sum- mation}

There are further applications of Ramanujan summation in the perturbative expansions of quantum field theory, which

\footnotetext{
xviii We refer to the van der Waals force in the narrow sense, also known as London-van der Waals force, i.e. the attractive multipole interaction between molecules [35].

xix On the other hand, Ref. [35] predicted a repulsive Casimir-van der Waals-type force, which agrees with an experiment with interacting materials immersed in a fluid [39].

$x x$ For interacting quantum field theories, renormalization involves more than subtracting a divergent term, which was sufficient in Sec. 4 for the free electromagnetic field. In the interacting case, one assigns renormalized values to the fields and their couplings, which are in general energy-scale dependent.
} 
can be treated by the $\zeta$-function regularization [47]. It is an alternative to dimensional regularization, which is most popular in perturbation theory. The $\zeta$-function regularization removes from the beginning the UV divergent terms in the Laurent series by inserting $\zeta(-k)$, thus preventing the necessity of counterterms. ${ }^{x x i}$ Stephen Hawking advocated its application in curved space-time [49].

Applications of the $\zeta$-function in bosonic string theory are reviewed in Refs. [50]. Ref. [51] summarizes a key point as follows: a particle mass $m$ is obtained as

$$
m^{2}=\frac{1}{\sigma}\left(j+\frac{D-2}{2} \sum_{k \geq 1}^{(\mathrm{R})} k\right),
$$

where $\sigma$ is the string tension, $D$ is the space-time dimension (such that a worldsheet lives in $D-2$ dimensions), and $j$ is the string excitation number (here also the Planck scale is set to 1$)$. The term with the sum over the modes $k$ represents the ground state energy $E_{0}$, where one applies Ramanujan summation, $E_{0}=-(D-2) / 24 \sigma$. The case $j=1$ describes spin-1 particles with only two polarization states, which must therefore be massless. This condition yields the space-time dimension $D=26$, where bosonic string theory is formulated [50]. The deeper reason is the requirement to cancel the conformal anomaly. A detailed pedagogical description is given in Ref. [52].

\section{Appendix}

\section{A. The failure of partial sums}

Many controversial discussions about relations like (3) - for instance, numerous comments on Ref. [12] - refer to partial sums of a few summands. In the framework of divergent series, separating them is conceptually wrong and leads to contradictions. It is entertaining to look at some examples, to see what one should beware of, e.g.,

$$
\begin{aligned}
\mathcal{R} & =1+(2+3+4)+(5+6+7)+(8+9+10)+\ldots \\
& \stackrel{?}{=} 1+9 \mathcal{R} \rightarrow \mathcal{R} \stackrel{?}{=}-1 / 8,
\end{aligned}
$$

which deviates from Ramanujan's value. One might even feel tempted to pay attention to this alternative value, since it is consistent with blocks of any odd number $u \geq 3$ of summands,

$$
\begin{aligned}
\mathcal{R} & =1+2+\cdots+\frac{u-1}{2}+\left(\frac{u+1}{2}+\cdots+\frac{3 u-1}{2}\right) \\
& +\left(\frac{3 u+1}{2}+\cdots+\frac{5 u-1}{2}\right)+\ldots \stackrel{?}{=} \frac{u^{2}-1}{8} \\
& +\mathcal{R} u^{2} \rightarrow \quad \mathcal{R} \stackrel{?}{=}-\frac{1}{8} .
\end{aligned}
$$

However, we can show that this approach is even intrinsically inconsistent by choosing blocks of an even number of $g$ summands (where the boundary terms are equally divided between the blocks),

$$
\begin{aligned}
\mathcal{R} & =1+2 \ldots\left(\frac{g}{2}-1\right)+\frac{g}{4}+\left(\frac{g}{4}+\left[\frac{g}{2}+1\right] \ldots\right. \\
& \left.+\left[\frac{3 g}{2}-1\right]+\frac{3 g}{4}\right)+\left(\frac{3 g}{4}+\left[\frac{3 g}{2}+1\right] \ldots\right)+\ldots \\
& \stackrel{?}{=} \frac{g^{2}}{8}+\mathcal{R} g^{2} \rightarrow \mathcal{R} \stackrel{?}{=}-\frac{1}{8} \frac{g^{2}}{g^{2}-1}
\end{aligned}
$$

which only coincides with the claim (A.2) in the limit $g \rightarrow$ $\infty$.

Of course, the applicability of partial sums can be disproved more easily, e.g., in Grandi's series or Dirichlet's $\eta$ function, if we write them as

$$
\begin{aligned}
\mathcal{G} & =(1-1)+(1-1)+(1-1)+\ldots \\
& =1+(-1+1)+(-1+1)+\ldots \\
\mathcal{E} & =(1-2)+(3-4)+(5-6)+\ldots \\
& =1+(-2+3)+(-4+5)+\ldots
\end{aligned}
$$

which seems to suggest the contradictory values $\mathcal{G} \stackrel{?}{=} 0$ or $\mathcal{G} \stackrel{?}{=} 1$, and $\mathcal{E} \stackrel{?}{=} \mp(1+1+1+1 \ldots) \doteq \mp \mathcal{C}$. Again we encounter the series $\mathcal{C}$, which also appeared in Ramanujan's Second Notebook [10], as we anticipated in Eq. (18). We will come back to it in Appendix B. In that case, a division into blocks of $n$ summands seems to suggest $\mathcal{C}=$ $(1+\cdots+1)+(1+\cdots+1)+\ldots \stackrel{?}{=} n \mathcal{C}, \mathcal{C} \stackrel{?}{=} 0$ or $\infty$ (while separating $k$ summands $\mathcal{C}=k+\mathcal{C}, \mathcal{C}=\infty)$.

\section{B. Analytic continuation from the unit disk}

We now follow the scheme of Sec. 2 by writing the series under consideration in terms of a variable $z \in \mathbb{C}$, such that they converge for $|z|<1$, and the divergent series of interest corresponds to the limit $z \rightarrow-1$. Working in the convergence region $|z|<1$ avoids, for instance, the confusion with partial sums. First, we repeat the geometrical series and Dirichlet's $\eta$-function,

$$
\begin{aligned}
& G(z)=1+z+z^{2}+z^{3}+\ldots \\
& =\frac{1}{1-z} \stackrel{z \rightarrow-1}{\longrightarrow} \mathcal{G}=1-1+1-1 \ldots \triangleq \frac{1}{2},
\end{aligned}
$$




$$
\begin{aligned}
\eta(z) & =1+2 z+3 z^{2}+4 z^{3}+\cdots=G(z)^{2}=G^{\prime}(z) \\
& =\frac{1}{(1-z)^{2}} \stackrel{z \rightarrow-1}{\longrightarrow} \mathcal{E}=1-2+3-4 \ldots \triangleq \frac{1}{4}
\end{aligned}
$$

As long as this limit is finite, taking the analytic continuation is trivial. This allows us to perform operations, which remain valid in the limit $z \rightarrow-1$, like

$$
\begin{aligned}
\eta(z)+G(z) & =\frac{1}{z}[\eta(z)-1] \\
& =2+3 z+4 z^{2}+\ldots \stackrel{z \rightarrow-1}{\longrightarrow} \frac{3}{4} \\
\eta(z)-G(z) & =z \eta(z) \\
& =z+2 z^{2}+3 z^{3}+\ldots \stackrel{z \rightarrow-1}{\longrightarrow}-\frac{1}{4} .
\end{aligned}
$$

In this framework, the limit $z \rightarrow-1$ is well controlled.

This is not obvious anymore when we deal with the pole of the geometrical series at $z=1$, in particular when we refer to the series

$$
\mathcal{C}=1+1+1+1+1 \ldots
$$

which worried us before in Sec. 2 and Appendix A. Here we consider three different regularizing functions (at $|z|<1$ ),

$$
\begin{aligned}
& C_{1}(z)=1-z+z^{2}-z^{3}+z^{4} \cdots=\frac{1}{1+z}, \\
& C_{2}(z)=1+z^{2}+z^{4}+z^{6} \ldots=\frac{1}{1-z^{2}}, \\
& C_{3}(z)=-\left(z+z^{3}+z^{5}+\ldots\right)=-\frac{z}{1-z^{2}} .
\end{aligned}
$$

We can involve the functions $C_{i}(z)$ in a variety of relations, such as $G(z)=(1+z) C_{2}(z)=1-(1+z) C_{3}(z)$. Of course, they work both in the form of series and of functions, in agreement with $C_{i}(z \rightarrow-1) \rightarrow \infty$. We can also build linear combinations of the functions $C_{i}(z)$, for instance,

$$
G(z)=C_{1}(z)-2 C_{3}(z)=\frac{1}{1-z},
$$

where the singularity at $z=-1$ is removed. When we now insert $\mathcal{C}=C_{1}(z \rightarrow-1)=C_{3}(z \rightarrow-1)$, and treat it as a finite constant, we obtain the finite value, which indeed matches $\zeta(0)$, as we saw in Eq. (18), and which Ramanujan had reported [10],

$$
\mathcal{C} \triangleq-\mathcal{G} \triangleq-\frac{1}{2}=\zeta(0)
$$

It was (apparently) a step of this kind that Ramanujan performed to compute the famous series (14), $\mathcal{R}=1+2+3+$ $4+5 \ldots$. Here we consider the regularizations

$$
\begin{aligned}
R_{1}(z) & =1-2 z+3 z^{2}-4 z^{3}+5 z^{4} \cdots=C_{1}(z)^{2} \\
& =-C_{1}^{\prime}(z)=\frac{1}{(1+z)^{2}} \\
R_{2}(z) & =1+2 z^{2}+3 z^{4}+4 z^{6} \ldots \\
& =\eta\left(z^{2}\right)=\frac{1}{\left(1-z^{2}\right)^{2}} .
\end{aligned}
$$

We introduced $R_{1}(z)$ before in Eq. (15), and we used it, at the regularized level, in identity (16), which we can now write in the compact form

$$
\eta(z)=R_{1}(z)+4 z R_{2}(z) .
$$

Again the singularity at $z=-1$ cancels on the right-hand side: note the an expansion in $\varepsilon=z+1$ leads to different Laurent series for $R_{1}(\varepsilon)=1 / \varepsilon^{2}$ and $R_{2}(\varepsilon)=(1+\varepsilon+$ $\left.3 \varepsilon^{2} / 4\right) / 4 \varepsilon^{2}+\mathcal{O}(\varepsilon)$, such that the right-hand side of Eq. (B.8) takes the expected form $1 / 4+\mathcal{O}(\varepsilon)$.

If we insert $\mathcal{R}=R_{1}(-1)=R_{2}(-1)$, and treat $\mathcal{R}$ as a finite constant, we retrieve Ramanujan's famous result

$$
\frac{1}{4} \stackrel{\wedge}{=}-3 \mathcal{R} \quad \Rightarrow \quad \mathcal{R} \triangleq-\frac{1}{12}=\zeta(-1)
$$

However, this procedure only works when the terms are arranged such that the limit of interest $(z \rightarrow-1$ in our case) is regular, otherwise, this step is not controlled. Consider, for instance the identity

$$
\begin{aligned}
R_{1}(z)+C_{1}(z) & =2-3 z+4 z^{2}-5 z^{3} \ldots \\
& =\frac{1}{z}\left[1-R_{1}(z)\right] .
\end{aligned}
$$

If we now insert $\mathcal{C}=C_{1}(-1)$ and $\mathcal{R}=R_{1}(-1)=$ $-\left.(1 / z) R_{1}(z)\right|_{z=-1}$, we end up with $\mathcal{C} \stackrel{?}{=}-1$, which contradicts Eq. (B.6).

Another example, which refers to $\mathcal{R}$, is the identity

$$
R_{2}(z)=R_{1}(z) \eta(z)=\frac{1}{(1+z)^{2}(1-z)^{2}}
$$

Carelessly inserting $\mathcal{R}=R_{1}(-1)=R_{2}(-1)$ purports $\mathcal{R} \stackrel{?}{=}(1 / 4) \mathcal{R}, \mathcal{R} \stackrel{?}{=} 0$ or $\infty$. The reason for this fiasco is that Eqs. (B.10) and (B.11) are singular at $z=-1$.

Ramanujan had either the right intuition to pick an appropriate relation, (16) or (B.8), where this step works, or it is based on additional considerations on his slate, which are not documented. It seems that he hardly knew any literature about the $\zeta$-function, but he rediscovered correct values of its analytic continuation to correct values of its analytic continuation to $\zeta(0), \zeta(-1)$, and $\zeta(-3)$, which cannot be by accident. In particular, he must have observed [10] the agreement of the results that he obtained in this way with the finite term in the series that we call Euler-Maclaurin expansion, cf. Sec. 3 . 


\section{The Riemann $\zeta$-function}

We have seen that a naive ansatz for the continuation can be plagued by subtleties when we hit a pole. An unambiguous approach to evaluate series like $\mathcal{C}$ and $\mathcal{R}$, as well as the cubic series (4), combines the terms such that - in the limit of interest - the singularity is removed, as it is done in the approaches of Eqs. (16), (B.8), and of Eqs. (18), (19), or by subtracting the corresponding integral: the result, given in Eq. (28), coincides with $\zeta(-k), k \in \mathbb{N}_{0}$.

The underlying concept is analytic continuation: if a complex function $f(z) \in \mathbb{C}, z \in \mathbb{C}$, is holomorphic in some region, then its analytic continuation is unique. Hence the existence of a complex derivative is a powerful property: a plausibility argument is that such a map $f(z)$, with $f^{\prime}(z) \neq 0$, is angle-preserving, cf. footnote [vii], which constrains the analytic continuation to a single possibility.

Being a pioneer in this field, Riemann extended the $\zeta$ function from the region with $\operatorname{Re} z>1$ (where it is defined by the convergent series (5)) to $\mathbb{C}-\{1\}$ by means of relations [14], which can be condensed into the functional equation

$$
\zeta(z)=\frac{(2 \pi)^{z}}{\pi} \sin \left(\frac{\pi z}{2}\right) \Gamma(1-z) \zeta(1-z),
$$

which is valid all over $\mathbb{C}$. We read off $\zeta(-1)=$ $\left(2 \pi^{2}\right)^{-1}(-1) \pi^{2} / 6=-1 / 12$, where we inserted $\Gamma(n)=$ $(n-1) !, n \in \mathbb{N}_{+}$, as well as Euler's Basel formula $\sum_{n \geq 1} 1 / n^{2}=\pi^{2} / 6$. Similarly we obtain $\zeta(-3)=$ $6 \zeta(4) /\left(8 \pi^{4}\right)=1 / 120$, by employing Euler's result $\zeta(4)=$ $\pi^{4} / 90$. This confirms again the Ramanujan summations (3) and (4). We further see that $\zeta(-2 n)=0, \forall n \in \mathbb{N}$ (due to the sin-function), in agreement with Eq. (28). ${ }^{x x i i}$ Finally we observe a simple pole at $z=1$, with the residue $\lim _{z \rightarrow 1}(z-$ 1) $\zeta(z)=1$, which is consistent with $\zeta(0)=-(1 / 2)=\mathcal{C}$.

The validity of a series representation of $\zeta(z)$, which converges all over $\mathbb{C}-\{1\}$, was demonstrated by Helmut Hasse [53]. This formula uses Eq. (7) and a double sum for $\eta(z)$,

$$
\begin{aligned}
\zeta(z) & =\frac{1}{1-2^{1-z}} \sum_{n \geq 0} \frac{1}{2^{n+1}} \\
& \times \sum_{k=0}^{n}(-1)^{k}\left(\begin{array}{c}
n \\
k
\end{array}\right) \frac{1}{(k+1)^{z}} .
\end{aligned}
$$

For $z=0$ the sum over $k$ corresponds to $(1-1)^{n}=\delta_{n, 0}$, and we confirm $\zeta(0)=-1 / 2$. Similarly, for $z=-1$ the second sum yields $\delta_{n, 0}-\delta_{n, 1}$, and we obtain once more $\zeta(-1)=-1 / 12$.

So could we have directly quoted these values and skipped the previous consideration? There are two reasons against it: we would not capture the magic of Ramanujan's way of thinking, and we would have missed the physical picture, which leads to the values of $\zeta(-n), n \in \mathbb{N}$. This picture justifies their application to the Casimir effect as a basic example of renormalization.

\section{Acknowledgement}

I would like to thank Kimball Milton for instructive comments. This work was supported by UNAM-DGAPAPAPIIT, grant number IG100219. 
1. https://mathworld.wolfram.com/

2. G. S. Carr, A Synopsis of Elementary Results in Pure and Applied Mathematics: Containing Propositions, Formulae, And Methods Of Analysis, With Abridged Demonstrations (C. F. Hodgson and Son, London, 1886).

3. R. Kanigel, The Man Who Knew Infinity: A Life of the Genius Ramanujan (Charles Scribner's Sons, 1991). K. Srinivas Rao, Srinivasa Ramanujan: A Mathematical Genius (East West Book Pvt Ltd, 1998).

4. G. S. Hardy, S. Ramanujan F.R.S., Nature 105 (1920) 494, https://doi.org/10.1038/105494a0.

5. http://ramanujan.sirinudi.org/html/ published_papers.html.

6. G. H. Hardy and S. Ramanujan, Asymptotic Formulæ in Combinatory Analysis, Proc. London Math. Society 17 (1918) 75, https://doi.org/10.1112/plms/s2-17.1.75

7. S. Ramanujan, Modular equations and approximations to $\pi$, Quart. J. Math. 45 (1914) 350, https://doi.org/10. $2307 / 2325206$

8. A. Robinson, Film: In search of Ramanujan, Nature 531 (2016) 576, https://doi.org/10.1038/531576a

9. https://www.qedcat.com/misc/ramanujans. letter.jpg

10. S. Ramanujan, Second Notebook (unpublished), Chapter VI. \{authentically reproduced in Ref. [11], Chapter 8\}.

11. B. Candelpergher, Ramanujan summation of divergent series (Lectures Notes in Mathematics, 2185 [hal-01150208v2], 2017).

12. https://www.youtube.com/watch?list= PLmNp3NTX4KXIqt jt2AKOr4DUIxn 64xj8G \\&V= jcKRGpMiVTw

13. R. Ayoub, Euler and the Zeta Function, Am. Math. Mon. 81 (1974) 1067, https://doi.org/10.2307/2319041

14. B. Riemann, Ueber die Anzahl der Primzahlen unter einer gegebenen Grösse, in Monatsberichte der Berliner Akademie, November 1859. A detailed discussion is included in: H. M. Edwards, Riemann's Zeta Function (Dover, 2001).

15. J. J. Gray, Bernhard Riemann, in Encyclopedia Britannica, https://www.britannica.com/biography/ Bernhard-Riemann

16. M. du Sautoy, The Music of the Primes: Searching to Solve the Greatest Mystery in Mathematics (HarperCollins, 2003).

17. D. J. Struik, A Concise History of Mathematics, 4th edition (Dover Publications, 1987).

18. M. E. Peskin and D. V. Schroeder, An Introduction to Quantum Field Theory (Addison-Wesley Advanced Book Program, 1995). A. Zee, Quantum Field Theory in a Nutshell (Princeton University Press, 2010). M. Kachelriess, Quantum Fields: From the Hubble to the Planck Scale (Oxford University Press, 2018).

19. W. Bietenholz, What are Elementary Particles? From Dark Energy to Quantum Field Excitations, Rev. Cub. Fis. 37 (2020) 146, arXiv:2011.07719 [physics.pop-ph].
20. H. B. G. Casimir and D. Polder, The Influence of Retardation on the London-van der Waals Forces, Phys. Rev. 73 (1948) 360, https://doi.org/10.1103/PhysRev.73.360

21. H. B. G. Casimir, On the attraction between two perfectly conducting plates, Proc. Kon. Ned. Akad. Wetensch. B 51 (1948) 793.

22. T. M. Apostol, An Elementary View of Euler's Summation Formula, Am. Math. Mon. 106 (1999) 409, https : / /doi .org/ $10.2307 / 2589145$

23. S. Ramanujan, Some properties of Bernoulli's numbers, J. Indian Math. Soc. 3 (1911) 219.

24. E. M. Lifshitz, The Theory of Molecular Attractive Forces between Solids, Soviet Phys. JETP 2 (1956) 73.

25. C. D. Fosco, F. C. Lombardo and F. D. Mazzitelli, Vacuum fluctuations and generalized boundary conditions, Phys. Rev. D 87 (2013) 105008, https://doi.org/10.1103/ PhysRevD.87.105008. B. A. Juárez-Aubry and R. Weder, Quantum field theory with dynamical boundary conditions and the Casimir effect, arXiv:2004.05646 [hep-th]; Quantum field theory with dynamical boundary conditions and the Casimir effect II: Coherent states, Phys. A 54 (2021) 105203, https: //doi.org/10.1088/1751-8121/abdecf

26. S. K. Lamoreaux, Demonstration of the Casimir Force in the 0.6 to $6 \mu \mathrm{m}$ Range, Phys. Rev. Lett. 78 (1997) 5, https: //doi.org/10.1103/PhysRevLett.78.5.

27. U. Mohideen and A. Roy, Precision Measurement of the Casimir Force from 0.1 to $0.9 \mu \mathrm{m}$, Phys. Rev. Lett. 81 (1998) 4549, https://doi.org/10.1103/ PhysRevLett.81.4549

28. G. Bressi, G. Carugno, R. Onofrio and G. Ruoso, Measurement of the Casimir Force between Parallel Metallic Surfaces, Phys. Rev. Lett. 88 (2002) 041804, https : / / doi . org/ 10 . 1103/PhysRevLett.88.041804

29. S. Weinberg, The cosmological constant problem, Rev. Mod. Phys. 61 (1989) 1, https://doi.org/10.1103/ RevModPhys.61.1. K. A. Milton, The Casimir effect: Physical manifestatios of zero-point energy (World Scientific, 2001). L. Feng, J. March-Russell, S. Sethi and F. Wilczek, Saltatory Relaxation of the Cosmological Constant, Nucl. Phys. B 602 (2001) 307, DOI: 10.1016/ S0550-3213(01)00097-9

P. J. E. Peebles and B. Ratra, The Cosmological Constant and Dark Energy, Rev. Mod. Phys. 75 (2003) 559, https: //doi.org/10.1103/RevModPhys.75.559

30. S. M. Carroll, The Cosmological Constant, Living Rev. Rel. 4:1 (2001) 1, https://doi.org/10.12942/ 1rr-2001-1

31. V. Sahni and A. Starobinsky, The Case for a Positive Cosmological $\Lambda$-term, Int. J. Mod. Phys. D 9 (2000) 373, https: //doi.org/10.1142/S0218271800000542

32. A. Riess et al., Observational Evidence from Supernovae for an Accelerating Universe and a Cosmological Constant, Astron. J. 116 (1998) 1009, https://doi.org/10.1086/ 300499. S. Perlmutter et al., Measurement of $\Omega$ and $\Lambda$ from 42 High-Redshift Supernovae, Astrophys. J. 517 (1999) 565, https://doi.org/10.1086/307221 
33. J. Schwinger, Casimir effect in source theory, Lett. Math. Phys. 1 (1975) 43, https://doi.org/10.1007/ BF00405585 J. Schwinger, J. DeRaad and K. A. Milton, Casimir Effect in Dielectrics, Ann. Phys. (N.Y.) 115 (1978) 1, https://doi.org/10.1016/0003-4916(78) 90172-0

34. K. Scharnhorst, On propagation of light in the vacuum between plates, Phys. Lett. B 236 (1990) 354, https: //doi.org/10.1016/0370-2693(90) 90997-K] [Erratum: Phys. Lett. B 787 (2018) 204, https://doi.org/ $10.1016 / j \cdot$ physletb.2018.12.002/.

35. I. E. Dzyaloshinskii, E. M. Lifshitz and L. P. Pitaevskii, General theory of van der Waals' forces, Sov. Phys. Uspekhi 4 (1961) 153, https://doi.org/10.1070/ PU1961v004n02ABEH003330

36. R. L. Jaffe, The Casimir Effect and the Quantum Vacuum, Phys. Rev. D 72 (2005) 021301, https : / doi.org/10.1103/ PhysRevD.72.021301

37. T. H. Boyer, Quantum Electromagnetic Zero-Point Energy of a Conducting Spherical Shell and the Casimir Model for a Charged Particle, Phys. Rev. 174 (1968) 1764, https:// doi.org/10.1103/PhysRev.174.1764

38. S. G. Mamaev and N. N. Trunov, Dependence of the vacuum expectation values of the energy-momentum tensor on the geometry and topology of the manifold, Theor. Math. Phys. 38 (1979) 228, https://doi.org/10. 1007/BF01018540 J. Ambjørn and S. Wolfram, Properties of the vacuum. I. Mechanical and thermodynamic, $A n$ nals Phys. 147 (1983) 1, https://doi.org/10.1016/ 0003-4916(83) 90065-9.

39. J. N. Munday, F. Capasso and V. A. Parsegian, Measured longrange repulsive Casimir-Lifshitz forces, Nature 457 (2009) 170, https://doi.org/10.1038/nature07610

40. M. P. Hertzberg, R. L. Jaffe, M. Kardar and A. Scardicchio, Attractive Casimir Forces in a Closed Geometry, Phys. Rev. Lett. 95 (2005) 250402, https://doi.org/10.1103/ PhysRevLett.95.250402

41. R. M. Cavalcanti, Casimir force on a piston, Phys. Rev. D 69 (2004) 065015, https://doi.org/10.1103/ PhysRevD.69.065015

42. P. van Baal, The QCD vacuum, Nucl. Phys. Proc. Suppl. 63 (1998) 126, https://doi.org/10.1016/ S0920-5632(97)00704-4.
43. G. Bhanot, W. Fischler and S. Rudaz, A multipole expansion and the Casimir-Polder effect in quantum chromodynamics, Nucl. Phys. B 155 (1979) 208, https : / / doi . org/10. 1016/0550-3213(79) 90363-8

44. F. Canfora and L. Rosa, Casimir energy in the GribovZwanziger approach to QCD, Phys. Rev. D 88 (2013) 045025, https://doi.org/10.1103/PhysRevD . 88 . 045025

45. O. Panella and A. Widom, Casimir effects in gravitational interactions, Phys. Rev. D 49 (1994) 917, https: / / doi.org/ 10.1103/PhysRevD.49.917 J. Q. Quach, Gravitational Casimir Effect, Phys. Rev. Lett. 114 (2015) 081104, https : //doi.org/10.1103/PhysRevLett.114.081104. [Erratum Phys. Rev. Lett. 118 (2017) 139901, https: //doi.org/10.1103/PhysRevLett.118.139901].

J. Hua and $\mathrm{H}$. Yu, Gravitational Casimir-Polder effect, Phys. Lett. B 767 (2017) 16, https://doi.org/10.1016/j. physletb.2017.01.038

46. F. Pinto, Gravitational Casimir effect, the Lifshitz theory, and the existence of gravitons, Class. Quant. Grav. 33 (2016) 237001, https://doi.org/10.1088/0264-9381/ $33 / 23 / 237001$

47. E. Elizalde, S. D. Odintsov, A. Romeo, A. A. Bytsenko and S. Zerbini, Zeta Regularization Techniques with Applications (World Scientific, 1994).

48. G. H. Hardy and J. E. Littlewood, Contributions to the Theory of the Riemann Zeta-Function and the Theory of the Distribution of Primes, Acta Mathematica 41 (1916) 119, https: //doi.org/10.1007/BF02422942

49. S. W. Hawking, Zeta function regularization of path integrals in curved spacetime, Commun. Math. Phys. 55 (1977) 133, https://doi.org/10.1007/BF01626516.

50. J. Polchinski, String Theory: Volume 1, An Introduction to the Bosonic String (Cambridge Monographs on Mathematical Physics, 1998). F. Toppan, Lecture Notes: String Theory and Zeta-function, CBPF-MO-002/00, http: //www . cbpf . br/ toppan/mo00200.pdf

51. M. D. Schwartz, Quantum Field Theory and the Standard Model, Section 15.3.5 (Cambridge University Press, 2014).

52. B. Zwiebach, A First Course in String Theory, Section 12.4 (Cambridge University Press, 2004).

53. H. Hasse, Ein Summierungsverfahren für die Riemannsche Zeta-Reihe, Math. Z. 32 (1930) 458, https://doi.org/ $10.1007 / \mathrm{BF} 01194645$ 